\title{
Du Québec vers les États-Unis : l'étude des lieux d'origine
}

\section{Bruno Ramirez et Jean Lamarre}

Volume 38, numéro 3, hiver 1985

Population et histoire

URI : https://id.erudit.org/iderudit/304285ar

DOI : https://doi.org/10.7202/304285ar

Aller au sommaire du numéro

Éditeur(s)

Institut d'histoire de l'Amérique française

ISSN

0035-2357 (imprimé)

1492-1383 (numérique)

Découvrir la revue

Citer cette note

Ramirez, B. \& Lamarre, J. (1985). Du Québec vers les États-Unis : l'étude des lieux d'origine. Revue d'histoire de l'Amérique française, 38(3), 409-422.

https://doi.org/10.7202/304285ar d'utilisation que vous pouvez consulter en ligne.

https://apropos.erudit.org/fr/usagers/politique-dutilisation/ 


\title{
NOTES DE RECHERCHE \\ DU QUÉBEC VERS LES ÉTATS-UNIS: L'ETUDE DES LIEUX D'ORIGINE*
}

\author{
BRUNO RAMIREZ \\ JEAN LAMARRE \\ Département d' histoire \\ Université de Montréal
}

Les historiens qui s'intéressent à l'histoire de l'immigration consacrent de plus en plus d'attention à l'étude des conditions socioéconomiques des pays et régions d'origine. Cette tendance est encore plus visible aux États-Unis où le nombre d'historiens de l'immigration a considérablement augmenté et où ce champ d'étude est sur le point de devenir partie intégrante de l'histoire sociale.

Cet intérêt à l'égard des pays ou régions d'émigration est loin d'être le reflet d'un purisme empirique. Au contraire, l'étude des lieux de provenance constitue un pas important dans la démarche visant à une meilleure compréhension du phénomène migratoire. Ainsi, à l'intérieur du processus global d'émigration, l'immigrant agit non seulement comme acteur historique se heurtant aux exigences de la société d'accueil au moment de son insertion, mais aussi comme un individu qui quitte un lieu précis en réponse à des besoins particuliers et sous des conditions particulières. L'hypothèse liée à notre démarche soutient qu'une analyse qui se limite à n'étudier l'émigration qu'à partir du moment où le migrant traverse la frontière du pays de destination tronque l'étude du phénomène migratoire, car il renferme, selon nous, une réalité spatiale beaucoup plus vaste, qui repose sur un ensemble de relations socio-économiques impliquant autant le lieu de départ que le lieu de destination. D'autant plus que le phénomène migratoire a souvent donné lieu à des mouvements de retour (〈return migration〉) permettant de canaliser des capitaux et du 〈know-how〉 qui sans doute ont influé sur l'évolution de la société d'origine.

* Remerciements à Mme Diane Courchesne, du Palais de Justice de Joliette et à Monique Montbriand, archiviste de l'Archevêché de Montréal pour leur précieuse collaboration. Nous tenons également à remercier Denise Helley, José Igartua, Yolande Lavoie, Paul-André Linteau, Anthony Masi, Jean-Claude Robert et Jacques Rouillard pour leurs commentaires et suggestions dont plusieurs n'ont pu être retenus dans cette version du texte. 
$\mathrm{Au}$ fil de leurs recherches, les historiens tentent de répondre à la question suivante: Qui étaient les immigrants?, confiants que les réponses apportées puissent aider à mieux comprendre les itinéraires socioéconomiques et culturels suivis par les immigrants au cours du processus d'insertion au sein de la société d'accueil, ainsi que pendant leur réinsertion dans leur société d'origine.

Cette démarche méthodologique nécessite, entre autre, l'étude du processus de sélection qui s'opère dans une région donnée et qui fait en sorte que certains décident de quitter alors que d'autres demeurent. Par ailleurs, une connaissance de la structure économique de la région est également indispensable pour identifier les différentes composantes de la population locale et du marché du travail, de manière à déterminer le type d'individu susceptible de recourir à l'émigration et, enfin, pour savoir si un tel recours était perçu comme une stratégie à court ou à long terme. Ce type de recherche a conduit à une meilleure caractérisation de la population émigrante en terme de composition par âge et par sexe, par métier, par niveau d'éducation, etc.

L'utilisation de ces outils d'analyse a déjà abouti à une meilleure compréhension des différents types d'émigration (permanente, temporaire, saisonnière) et des changements qui ont pu y survenir d'une période à une autre ${ }^{1}$. Ainsi, des études récentes sur le mouvement d'émigration de l'Italie vers le Québec, par exemple, ont fait état de transformations subies par ce mouvement au cours de trois périodes historiques, soit 1870-1890, 1890-1910 et 1910-1929, transformations qui trouvent leurs origines dans les conditions mêmes qui prévalaient tant dans le pays d'origine que dans le pays de destination ${ }^{2}$.

Dans d'autres cas, les historiens ont réalisé des progrès importants en décelant certains modèles résidentiels d'un groupe d'immigrants donné et en rendant compte de leurs comportements dans le marché du travail et dans le marché matrimonial ${ }^{3}$.

I Voir l'article de John. S. MacDonald et Leatrice D. MacDonald, «Chain Migration, Ethnic Neighborhood Formation and Social Networks», Milbank Memorial Fund Quarterly, XLII (January 1964): 82-91. Et plus récemment, Josef Barton, Peasants and Strangers: Italians, Rumanians and Slovaks in an American City, 1890-1950. (Cambridge, Mass., Harvard University Press, 1975).

Bruno Ramirez, Les premiers Italiens de Montréal: l'origine de la Petite Italie du Québec (Montréal, Les Éditions du Boréal Express, 1984).

3 Virginia Yans-McLaughlin, Family and Community: Italian Immigrants in Buffalo, 1880 1930 (Ithaca, New York: Cornell University Press, 1977); John Briggs, An Italian Passage: Immigrants to Three American Cities, 1890-1930 (New Haven: Yale University Press, 1978); John Zucchi, «The Italian Immigrants of the St. John's Ward, 1875-1915: Patterns of Settlement and Neighbourhood Formation», Occasional Papers (Toronto: The Multicultural History Society of Ontario, 1981); Mauro Peressini, «Pratiques et stratégies migratoires: le cas des Italiens originaires du Frioul», Thèse de maîtrise, Département d'Anthropologie, Université de Montréal, 1983. Sylvie Taschereau complète en ce moment son mémoire de maítrise en Histoire (UQAM) portant sur la relation entre les lieux d'origine et les modèles maritaux chez les Italiens de Montréal. 
Mais, au-delà des contributions spécifiques de ces recherches historiques, il nous apparaît important de souligner que l'étude du phénomène migratoire doit considérer la question des lieux d'origine comme essentielle et l'insérer dans sa problématique comme élément central de recherche.

Si nous examinons l'historiographie du mouvement d'émigration des Canadiens français vers les Etats-Unis, l'absence de ce type de problématique est évidente. Les progrès importants réalisés par les historiens de l'économie et les démographes dans leurs recherches visant à 'mesurer l'ampleur de l'émigration des Canadiens français ${ }^{4}$ sont loin d'avoir été accompagnés par des progrès semblables du côté des recherches sur les aspects qualitatifs et régionaux de ce mouvement d'émigration. Ce n'est pas notre intention de faire ici une analyse critique de cette historiographie. Nous voulons simplement souligner le fait que nos connaissances sur les régions d'émigration au Québec, sur leurs caractéristiques structurelles et conjoncturelles ainsi que sur leurs modèles d'émigration demeurent jusqu'à ce jour fort limitées. La majorité des informations que nous possédons sont demeurées au stade des généralités. Nous savons depuis longtemps, par exemple, que la grande majorité de la population canadienne-française qui émigra provenait des régions rurales et que cet exode était directement lié à la crise agricole et au haut taux de natalité qui caractérisaient le Québec à cette époque. Cette affirmation est certainement vraie, mais, en même temps, trop générale pour nous permettre de comprendre les transformations que le mouvement d'émigration a pu subir au cours des années ainsi que de rendre possible une reconstitution des réseaux d'émigration qui relièrent certaines régions du Québec avec d'autres régions des ÉtatsUnis.

Cette lacune se réflète clairement dans les deux principales études réalisées sur le sujet depuis les quinze dernières années ${ }^{5}$. L'étude de Ralph Vicero, bien qu'elle contienne l'analyse la plus complète jamais produite sur le mouvement d'émigration des Canadiens français, fournit peu d'information sur la configuration régionale de ce mouvement. En analysant les «facteurs de répulsion» qui agissaient sur la Province, Vicero fait un certain nombre de références à plusieurs régions. Cepen-

4 Gilles Paquet, «L'émigration des Canadiens français vers la Nouvelle Angleterre, 18701910: prises de vues quantitatives», Recherches sociographiques, V, 3 (sept-déc. 1964): 319-370; Albert Faucher, «L'émigration des Canadiens français au XIX ${ }^{\mathrm{e}}$ siècle; position du problème et perspectives», Recherches sociographiques, V, 3, (sept-déc. 1964): 277-317; Yolande Lavoie, L'émigration des Canadiens aux États-Unis avant 1930. Mesure du phénomène (Montréal, PUM, 1972).

Ralph D. Vicero, «The Immigration of French-Canadians to New England, 1840-1900: A Geographical Analysis». Thèse de Doctorat, University of Wisconsin, 1968; Tamara K. Hareven, Family Time and Industrial Time: The Relationship Between the Family and Work in a New England Industrial Community (Cambridge, Mass.: Harvard University Press, 1982). 
dant, ces références sont, pour la plupart, tirées de sources contemporaines et ne sont pas analysées dans une perspective régionale. L'étude sur Manchester, New Hampshire, de Tamara Hareven constitue, sans aucun doute, la plus sophistiquée des recherches effectuées sur les Franco-Américains jusqu'à ce jour, tant au niveau de la méthodologie employée que du cadre théorique dans lequel elle s'insère. Un des principaux objectifs de T. Hareven est de montrer comment les réseaux de parenté qui furent transférés du Québec vers Manchester ont agi sur le processus d'adaptation au style de vie et de travail des immigrants canadiens-français, dans ce qui était l'un des plus importants centres industriels de la Nouvelle-Angleterre. Malgré la brillante analyse qu'elle fait de cette question, Hareven admet que les recherches dans ce domaine sont freinées par le manque de connaissances de la dynamique socioéconomique caractérisant les localités d'émigration. Ayant soulevé la question de savoir dans quelle mesure le type de liens de parenté présent dans une localité donnée au Québec pouvait se retrouver intact à Manchester, Hareven répond: «In the absence of a full-fledged comparison of family structure, demographic behavior, women's labor-force participation, and family economy for Manchester and the Quebec parishes of origin, it would be impossible to answer this question conclusively.» ${ }^{6}$ Un des problèmes que nous pourrions ajouter est celui d'identifier les différentes paroisses d'origine du Québec faisant partie d'un réseau d'émigration donné.

Quant aux récentes recherches en histoire des Franco-Américains, elles n'ont pas réellement permis d'ajouter de façon significative des données susceptibles de faire progresser nos connaissances des lieux d'origine ${ }^{7}$.

Il est vrai que plusieurs monographies sur les Franco-Américains, produites dans le sillage de ce renouveau d'intérêt, s'appuient sur une méthodologie plus élaborée et sur une utilisation plus sophistiquée des données historiques. Ces travaux ont contribué à une connaissance plus approfondie du processus d'intégration et d'adaptation au sein de la société d'accueil $^{8}$. Mais encore là, l'émigration des Canadiens français

6 Hareven, op. cit., 116.

La prise de conscience de cette lacune par les historiens travaillant dans ce champ d'étude s'est clairement manifestée lors du colloque «La situation de la recherche sur la Franco-Américanie» tenu au Collège Assumption à Worcester, Massachusetts, et dont les communications furent réunies dans un numéro spécial de la revue Vie française (Québec, 1980). Voir en particulier les remarques de Frances Early et de Daniel Walkowitz aux pages 26 et 36 .

8 En plus des travaux de Hareven, voir particulièrement Peter Haebler, «Habitants in Holyoke: The Development of the French-Canadian Community in a Massachusetts City, 1865-1910», thèse de Doctorat, University of New Hampshire, 1976; Frances H. Early, «French-Canadian Beginnings in an American Community: Lowell, Massachusetts, 1868-1886», thèse de Doctorat, Université Concordia, 1979; Daniel Walkowitz, Worker City, Company Town, Iron and CottonWorker Protest in Troy and Cohoes, New York (Urbana; University of Illinois Press, 1978); Pierre Anctil, «Aspects of Class Ideology in a New England Ethnic Minority: The Franco-Americans of Woonsocket, Rhode Island», thèse de Doctorat, New School for Social Research, New York, 1979. 
continue d'être étudiée à travers une série de généralisations qui nous empêchent de voir l'immigrant comme un acteur historique faisant partie d'une réalité locale précise et pour qui l'itinéraire social et économique a pu différer de celui qui prévalait en d'autres temps ${ }^{9}$.

La qualité des sources d'archives disponibles et des statistiques officielles d'immigration tant au Canada qu'aux États-Unis a pu constituer un facteur important dans le retard qui caractérise les recherches dans ce champ d'étude. Contrairement à d'autres pays, l'Italie par exemple, où des statistiques détaillées concernant la population émigrante sont compilées annuellement au niveau des municipalités, les statistiques canadiennes sur la population émigrante sont très générales, situation issue, au moins en partie, du peu d'intérêt porté par les autorités fédérales canadiennes face au mouvement d'émigration, et, aussi peut-être, de la perméabilité de la frontière entre le Canada et les ÉtatsUnis.

La situation n'est pas foncièrement différente lorsque l'on regarde les statistiques américaines. Les données des recensements fédéraux américains ainsi que ceux réalisés au niveau des États, constituent certes des sources essentielles pour l'étude du mouvement migratoire du Québec vers les États-Unis, tant au niveau quantitatif qu'au niveau de la composition ethnique de la population immigrante (une distinction est établie entre Canadiens français et Canadiens anglais). Cependant, ces sources perdent de leur valeur si on s'intéresse aux lieux d'origine (comté ou paroisse) de la population canadienne-française résidant aux ÉtatsUnis ${ }^{10}$. Même les listes nominatives des recensements passent sous

9 Il faut souligner ici que les études biographiques sur les immigrants ainsi que les études basées sur les «histoires de vie» peuvent fournir des informations utiles sur les lieux d'origine de ces immigrants. Deux exemples récents, ceux de Frances H. Early, «The Rise and Fall of Felix Albert: Reflections on the Aspirations of Habitant Immigrants to Lowell in the Late Nineteenth Century», dans Raymond Breton et Pierre Savard, ed., The Quebec and Acadian Diaspora in North America (Toronto: The Multicultural History Society of Ontario, 1982, 25-38, et Jacques Rouillard, Les derniers migrants: une enquête auprès de Québécois, 1900-1930 (à paraître aux Éditions du Boréal Express). Ajoutons que l'historiographie franco-américaine comprend quelques ouvrages qui nous fournissent des éléments utiles pour étudier les lieux d'origine en nous donnant les noms et les lieux de naissance de Canadiens français établis dans une paroisse ou une ville des États-Unis. Citons ici le livre de H.-A. Dubuque, Le guide canadien-français de Fall River et notes historiques sur les Canadiens de Fall River (Fall River, E. Lamoureux, 1888), 260 p., celui de U. Forget sur La paroisse St-Jean-Baptiste de Warren, État du Rhode Island, (1877-1952) (Montréal, Imprimerie Populaire Ltée, 1952), 395 p. ainsi que le livre de Marie-Louise Bonier sur Les débuts de la colonie franco-américaine de Woonsocket, Rhode Island (Framingham, Mass., Lakeview Press, 1920), 342 p. La monographie de U. Forget, particulièrement riche en données démographiques sur les Franco-Américains de Warren, constitue d'ailleurs une des sources majeures utilisées dans le cadre d'un mémoire de maîtrise présentement en cours; Jean Lamarre, "Étude d'une communauté canadienne-française de la Nouvelle-Angleterre: le cas de Warren, Rhode Island (1880-1895)», Université de Montréal.

${ }_{10}$ Pour un examen détaillé des problèmes soulevés par ces sources officielles, voir Yolande Lavoie, «Les mouvements migratoires des Canadiens entre leur pays et les États-Unis au XIX ${ }^{\mathrm{e}}$ et au XX ${ }^{\mathrm{e}}$ siècles: étude quantitative», Hubert Charbonneau, éd., La population du Québec: études rétrospectives (Montréal, Les Éditions du Boréal Express, 1973), 73-88. 
silence le lieu de naissance de la population recensée, mentionnant seulement le pays d'origine.

Compte tenu de cette situation, notre présente étude a pour but de pallier à certaines difficultés posées par la qualité des données officielles existantes, d'explorer la pertinence d'un certain nombre de sources qui peuvent aider le chercheur à mieux cerner la dimension régionale du mouvement d'émigration en provenance du Québec et à établir les liens entre ces régions d'origine et les lieux de destination. Notre discussion mettra l'accent sur un comté particulier d'émigration, le comté de Berthier.

Les registres de mariages constituent une des sources disponibles nous permettant d'identifier avec précision les lieux d'origine des émigrants canadiens-français aux États-Unis. Ces registres nous informent, entre autres, de la date du mariage, du nom des époux, de la date de leur naissance et du nom de la paroisse où ils sont nés. À moins d'avoir été perdus ou détruits, ces registres sont normalement conservés dans la paroisse où le mariage a été célébré. On peut également consulter les certificats de mariages correspondants qui, eux, sont conservés à l'hôtel de ville de la municipalité en question.

Il est évident que l'utilité de cette source est limitée seulement à une composante de la population immigrante, notamment, les hommes et les femmes né(e)s au Québec, qui ont quitté leur province en tant que célibataires et qui se sont marié(e)s dans une paroisse francoaméricaine.

De plus, cette source pose un problème interprétatif à cause de l'intervalle de temps entre la date de naissance (au Québec) et la date de mariage (aux Etats-Unis). Car bien que nous connaissions le lieu d'origine de l'immigrant en question, nous ignorons le moment exact où l'émigration s'est produite et si la personne émigre directement de son lieu de naissance vers son lieu de mariage. Ainsi, nous ne pouvons utiliser ces données pour établir une relation spatiale directe entre le lieu de naissance et le lieu de résidence aux États-Unis. Cependant, on ne peut douter de la validité de ces sources pour identifier certaines régions du Québec comme étant des centres majeurs d'émigration. En fait, nous pouvons considérer qu'une sur-représentation de certains comtés d'origine à l'intérieur de la population d'une paroisse francoaméricaine donnée peut suggérer l'existence d'un réseau de migration liant les deux localités en question. Par exemple, sur plus de 40 comtés du Québec représentés au sein des couples nés au Québec et mariés dans la paroisse de Sainte-Anne de Fall River, Mass., et de celle de Saint-Antoine de Manchester, N.H., le comté de Rimouski est le lieu d'origine de 23,5\% d'entre eux dans le premier cas, et de moins de $4 \%$ dans le deuxième cas. Ceci suggère l'existence d'une chaîne migratoire reliant le comté de Rimouski à la ville de Fall River. 
En explorant cette avenue de recherche, nous avons utilisé des copies des registres de mariages compilés par une société généalogique de la Nouvelle-Angleterre qui reproduit les informations contenues dans les registres originaux. Nous avons étudié la paroisse Sainte-Anne de Fall River, Mass., la paroisse Saint-Anthony et la paroisse Saint-JeanBaptiste, toutes deux de Manchester, N.H. Nous avons également eu recours au registre des décès de l'État du Rhode Island, compilés par la même société généalogique, et qui comprend des données sur 4357 décès de personnes d'origine canadienne-française (nées au Québec) (voir tableaux I et II). Même si le problème de l'intervalle de temps est encore plus évident dans ce cas-ci que dans celui des mariages, il nous permet néanmoins d'obtenir une représentation plus exacte des comtés d'origine, dans la mesure où il est question de toute la population d'ori-

TABLEAU I

Lieux de naissance des individus nés au Québec et mariés dans les paroisses Sainte-Anne de Fall River, Mass. et de Saint-Anthony, Manchester, New Hampshire.

\begin{tabular}{|c|c|c|c|c|}
\hline \multirow[b]{2}{*}{ Comtés } & \multicolumn{2}{|c|}{ Saint-Anne } & \multicolumn{2}{|c|}{ Saint-Anthony } \\
\hline & individus* & $\%$ & individus** & $\%$ \\
\hline Rimouski & 114 & 23,5 & & \\
\hline Nicolet & 30 & 6,2 & 88 & 11,3 \\
\hline Lotbinière & 37 & 7,6 & 56 & 7,3 \\
\hline Arthabaska & & & 54 & 6,9 \\
\hline Shefford & & & 54 & 6,9 \\
\hline Kamouraska & 28 & 5,8 & & \\
\hline Compton & & & 45 & 5,7 \\
\hline Hochelaga & 25 & 5,2 & & \\
\hline Mégantic & & & 36 & 4,6 \\
\hline Yamaska & & & 32 & 4,1 \\
\hline autres & $250^{1}$ & 51,6 & $414^{2}$ & 53,1 \\
\hline total & 484 & 100,0 & 779 & 100,0 \\
\hline
\end{tabular}

1. Moins de $4 \%$ et plus de $1 \%$ : Richelieu, Bagot, Yamaska, Montmagny, Compton, Shefford, Terrebonne, Charlevoix, Lévis, Iberville, Berthier, Stanstead, Arthabaska, Témiscouata, L'Islet, Québec, Rouville, Saint-Maurice, Joliette et Gaspé.

2. Moins de 4\% et plus de 1\%: Saint-Maurice, Rimouski, Stanstead, Wolfe, Hochelaga, Drummond, Beauce, Bagot, Richelieu, Richmond, Charlevoix, Québec, Lévis, Dorchester et Iberville.

* $80 \%$ de ces individus sont nés entre 1870 et 1899

** $79,2 \%$ de ces individus sont nés entre 1870 et 1909 .

Sources: E. Bisson and Sr. Pauline Giroux-Méthot, Repertory of Marriages 1899-1976 (Manchester, publié par Giroux-Méthot, 1977), 230 p. P.-H. La Ronde, L'état civil franco-américain (Manchester, De Henris Associates, 1951); R. J. Quintin, Sainte-Anne de Fall River, 1869-1930 (Pawtucket, R. I., publié par l'auteur, 1979), 333 p. 
TABLEAU II

Lieux de naissance des individus nés au Québec et décédés dans l'État du Rhode Island. Total: 4357.

\begin{tabular}{lcc}
\hline Comtés & $\begin{array}{c}\text { Nombre d'individus } \\
\text { nés au Québec }\end{array}$ & $\%$ \\
\hline Richelieu & 721 & 16,5 \\
Berthier & 589 & 13,5 \\
Bagot & 336 & 7,7 \\
Saint-Hyacinthe & 242 & 5,5 \\
Drummond & 235 & 5,4 \\
Yamaska & 234 & 5,3 \\
Joliette & 231 & 5,1 \\
Shefford & 189 & 4,3
\end{tabular}

Moins de $4 \%$ et plus de $1 \%$ : Maskinongé, Hochelaga, Iberville, Portneuf, Champlain, Lotbinière, Rouville, Saint-Maurice, Rimıuski, Brôme, Nicolet, Kamouraska, Chambly.

Sources: R. J. Quintin, Franco American Burials of Rhode Island, vol. I et II (Pawtucket, R. I., publié par l'auteur, sans date), $608 \mathrm{p}$.

gine canadienne-française du Rhode Island, comparativement à la population d'une seule paroisse pour le cas précédent ${ }^{11}$.

Il va sans dire que si nous appliquions systématiquement cette méthode à toutes les paroisses franco-américaines de la NouvelleAngleterre ou à un échantillon représentatif, nous pourrions reconstituer une carte approximative des nombreuses localités du Québec qui ont contribué à l'émigration des nôtres vers les États-Unis. Le fait d'identifier les localités d'exode et leur poids démographique au sein du mouvement global d'émigration ouvrirait de nouvelles et d'importantes pistes de recherches. Par exemple, l'analyse sociale, économique et démographique d'une région donnée au Québec acquerrait une dimension nouvelle et dynamique si elle était insérée dans le contexte de l'émigration.

De plus, la documentation locale peut servir de source très importante dans la mesure où elle nous permettrait de connaître la façon avec

11 Les registres américains de naturalisation constituent une autre source par laquelle on peut retracer les lieux d'origine des immigrants. Quand les immigrants voulaient acquérir la citoyenneté américaine, ils devaient fournir non seulement le nom de leur pays d'origine mais aussi la localité (ville ou village) où ils étaient nés. Il est évident que l'utilisation de cette source limite le chercheur à l'étude d'une catégorie spécifique d'immigrants, c'est-à-dire ceux qui ont délaissé leur statut pour devenir citoyen américain. De plus, cette source pose également le problème d'établir un lien géographique et temporel direct entre le lieu de naissance et le lieu de résidence lors de la demande de la naturalisation. Il faut souligner que les immigrants canadiensfrançais sont ceux qui retardent le plus longtemps le moment avant de demander leur naturalisation. Une monographie tirée du recensement américain indiquait que la durée moyenne de résidence avant qu'ils demandent leur naturalisation était de seize ans et demi. Voir Carpenter, Immigrants and Their Children (Washington, DC, 1927), 264. 
laquelle une localité en particulier (paroisse ou comté) a vécu l'expérience de l'émigration. Nous pensons à des sources telles les journaux, les biographies locales, l'histoire locale ainsi que les archives paroissiales et celles du comté. En explorant cette avenue, nous avons analysé systématiquement les Rapports pastoraux et les Actes notariés, deux sources appartenant aux archives locales. Pour notre étude, nous avons choisi d'examiner le comté de Berthier, comté qui a contribué d'une manière significative au mouvement d'émigration vers les États-Unis. En effet, l'examen des registres de décès de l'État du Rhode Island révèle que Berthier représente le comté d'origine du plus grand nombre de Canadiens français, même si, comme nous le verrons plus loin, le Rhode Island ne fut pas la seule destination.

Un rapide regard sur les recensements fédéraux canadiens permet d'obtenir une idée assez précise de l'importance de l'exode à partir du comté de Berthier. Durant les trois dernières décennies du XIX ${ }^{\mathrm{e}}$ siècle, période où l'exode a connu son apogée, la population de Berthier n'a connu aucune augmentation et a même accusé une baisse, passant de 19435 en 1871 à 18982 en $1901^{12}$. Si on considère que durant la même période le Québec voit sa population augmenter de $38,4 \%$, on peut mieux comprendre l'impact de l'émigration (tant à l'intérieur du Québec qu'à l'extérieur), sur la structure démographique de ce comté ${ }^{13}$.

Les Rapports pastoraux nous permettent par ailleurs de suivre le flot d'émigration entre les années de recensement et nous fournissent des informations additionnelles sur la composition de ce mouvement pour chacune des années. Dans leurs rapports annuels, les curés notaient le nombre de familles qui partaient vers les États-Unis ainsi que le nombre de jeunes adultes qui empruntaient le même chemin.

Malheureusement, pour la période que nous étudions, ces rapports ne furent pas soumis annuellement (ou s'ils le furent, ils ont été égarés). Par conséquent, les données que nous avons pu en tirer demeurent fragmentaires. Leur valeur en tant que source locale de l'émigration des Canadiens français n'en demeure pas moins précieuse. De toutes les paroisses composant le comté de Berthier, celle de Saint-Cuthbert fournit les rapports les plus complets. Les informations que nous avons compilées à partir de ces rapports (voir tableau III) font ressortir différentes caractéristiques du mouvement d'émigration issu de ce comté. Nous avons noté, par exemple, que malgré certaines fluctuations, le mouvement d'émigration vers les États-Unis n'a pas subi d'interruption. Nous pouvons également mieux calculer le poids démographique des départs en le comparant à la population totale de la paroisse. Ainsi en 1898, année record pour Saint-Cuthbert, sur 512 familles vivant dans

12 Recensement du Canada, 1871, vol. I, Tableau III: 1901, vol. I, Tableau VII.

M.C. Urquhart et K. Buckley, ed., Historical Statistics of Canada (Toronto, 1965), 14. 
cette paroisse, 35 ont pris le chemin des États-Unis. Les rapports démontrent aussi qu'au moment où l'émigration de type familial constituait la forme prédominante du phénomène migratoire, un nombre important de jeunes adultes prenaient eux aussi la route des États-Unis, alimentant ainsi ce mouvement d'une manière significative.

TABLEAU III

Population et émigration, 1881-1898.

Paroisse Saint-Cuthbert, comté de Berthier

\begin{tabular}{|c|c|c|c|c|c|}
\hline Année & Population & $\begin{array}{c}\text { Nombre } \\
\text { de familles }\end{array}$ & $\begin{array}{c}\text { Nombre } \\
\text { de familles } \\
\text { parties } \\
\text { aux É.-U. }\end{array}$ & $\begin{array}{c}\text { Nombre } \\
\text { de familles } \\
\text { revenues } \\
\text { des É.-U. }\end{array}$ & $\begin{array}{c}\text { Nombre } \\
\text { de jeunes } \\
\text { partis } \\
\text { aux É.-U. }\end{array}$ \\
\hline 1881 & 2764 & 490 & 14 & 11 & 40 \\
\hline 1882 & 2768 & 490 & 21 & 7 & 43 \\
\hline 1883 & ND & ND & ND & ND & ND \\
\hline 1884 & 2562 & ND & 11 & 6 & 48 \\
\hline 1885 & 2800 & 582 & 7 & 15 & 40 \\
\hline 1886 & 2825 & 512 & 22 & 3 & 28 \\
\hline 1887 & 2610 & 520 & 16 & 4 & 50 \\
\hline 1888 & 2780 & ND & 17 & 12 & 40 \\
\hline 1889 & 2789 & ND & 30 & 15 & 48 \\
\hline 1890 & ND & ND & ND & ND & ND \\
\hline 1891 & 2630 & ND & 19 & - & - \\
\hline 1892 & 2668 & 532 & 26 & 14 & 68 \\
\hline 1893 & 2580 & 520 & 24 & 6 & 40 \\
\hline 1894 & 2425 & 540 & 5 & 8 & 7 \\
\hline \multicolumn{6}{|l|}{1895 à } \\
\hline 1897 & ND & ND & ND & ND & ND \\
\hline 1898 & 2643 & 512 & 35 & 7 & 18 \\
\hline \multicolumn{3}{|c|}{ Moyenne annuelle, 1881-1898: } & 19 & 9 & 39 \\
\hline
\end{tabular}

Sources: Rapports pastoraux, Paroisse Saint-Cuthbert, Archevêché de Montréal. ND: Non déterminé.

Finalement, les rapports fournissent des informations sur le nombre de familles qui reviennent des États-Unis, permettant ainsi de mieux suivre le mouvement de va-et-vient de part et d'autre de la frontière, mouvement qui comme on le sait, constitue une des caractéristiques majeures du mouvement d'émigration des Canadiens français aux ÉtatsUnis. Dans le cas de Saint-Cuthbert, les données ne nous permettent pas d'établir avec précision le modèle de ce mouvement de va-et-vient. Néanmoins, une des conclusions que nous pouvons tirer de ce tableau est qu'à l'exception des années 1885 et 1894, le nombre de familles qui reviennent est beaucoup moins important que celui des familles qui quittent. 
Les actes notariés constituent l'autre source d'archives locales que nous avons utilisée pour les fins de cette recherche. Ces actes fournissent des informations sur une variété de transactions effectuées dans un comté donné, telles les ventes de terre ou autre propriété, quittances de dettes, obligations, octrois de procuration, testaments, etc. Dans la plupart des cas où une des deux parties en cause vivait aux États-Unis, l'acte en faisait mention. Cette donnée était souvent accompagnée d'informations supplémentaires concernant la profession des dites personnes et leur lieu de résidence aux États-Unis. Par exemple, l'acte no 825, signé par le notaire public H.-S. Champagne, le 15 juin 1882, concerne une transaction dans laquelle un dénommé Alexis Renault, ancien cultivateur de Saint-Damien et résidant à Holyoke, Massachusetts, a vendu par procuration, une terre située à Saint-Damien mesurant cinq arpents par vingt arpents pour la somme de $350 \$$. Dans un autre cas, un fermier de Saint-Cuthbert s'engage à verser annuellement un montant d'argent spécifié à titre de rente viagère à son frère qui est prêtre à Chicago. Autre exemple, l'acte no 8974 signé devant le notaire public F.-E. Rouleau, le 17 mai 1903, confirmait que Hermine Allard, demeurant à Woonsocket, Rhode Island, donnait, par voie de procuration, à Léandre Desfresne, cultivateur résidant à Berthier, le droit d'agir légalement en son nom afin qu'il prenne possession de la part d'héritage lui revenant à la suite du décès de ses parents. Une analyse de la nature de ces transactions et des circonstances qui les entourent peut nous éclairer sur le type de relation entretenue par les émigrants avec leur lieu d'origine, leur propriété, leur famille et amis ainsi qu'avec leur lieu de résidence aux Etats-Unis.

Pour notre recherche, nous avons répertorié tous les actes de vente de terre contenus dans les archives des notaires publics ayant oeuvré dans le comté de Berthier de 1875 à 1905. Seuls les actes de vente de terre dont le vendeur ou l'acheteur résidait aux États-Unis furent retenus.

Ces données sont résumées ici de façon à mettre en évidence deux aspects majeurs du phénomène migratoire, c'est-à-dire, les dimensions spatiale et temporelle. Ainsi, le tableau IV donne un excellent aperçu de l'éventail des destinations reliant le comté de Berthier aux différentes localités aux États-Unis. Évidemment, nous ne prétendons pas affirmer que les individus impliqués dans de telles transactions soient représentatifs de l'ensemble de la population de ce comté. Néanmoins, il serait difficile de prétendre, sur la base de la propriété terrienne, que les destinations choisies par les immigrants propriétaires de Berthier soient tout à fait différentes de celles préférées par les immigrants non-propriétaires du même comté.

Comme le tableau l'indique, les États du Rhode Island et du Massachusetts constituent, et de loin, les lieux de destination par excel- 
lence. Ensemble, ils recueillent $75 \%$ de toutes les destinations. Malgré la question de la représentativité déjà soulevée, ces données constituent, malgré tout, un point de départ intéressant pour l'étude des réseaux de migration. D'autres recherches pourront aider à établir avec plus de précision quand et comment ces réseaux se sont créés, à déterminer leur rôle dans l'expansion du mouvement migratoire ainsi qu'à soupeser l'attraction exercée par les localités du Rhode Island et du Massachusetts sur la population de Berthier.

TABLEAU IV

Lieux de résidence aux États-Unis des émigrants du Comté de Berthier, par État et pourcentage. 1875-1905

\begin{tabular}{lc}
\hline \multicolumn{1}{c}{ État } & $\%$ \\
\hline Rhode Island & 40,5 \\
Massachusetts & 35,3 \\
Michigan & 5,8 \\
Connecticut & 4,7 \\
New York & 4,2 \\
Moins de 3\%: New Hampshire, Minnesota, Vermont, Nevada, Montana, Illinois.
\end{tabular}

Compilé à partir des actes notariés du comté de Berthier, 1875-1905. Archives du Palais de Justice, Joliette.

Nous espérons que cette piste aidera à percevoir le mouvement d'émigration des Canadiens français en des termes beaucoup moins abstraits et généraux pour qu'enfin il soit perçu comme une expérience concrète et régionale, et comme un phénomène s'insérant dans un cadre géo-économique précis, impliquant autant le pays d'origine que le pays de destination.

Ces données nous aident également à obtenir une meilleure compréhension de la dimension temporelle du mouvement d'émigration. Comme le graphique I. le montre, les ventes de terre par les émigrants provenant du comté de Berthier et résidant en Nouvelle-Angleterre se produisent à chacune des trente années de la période étudiée. Il n'est pas dans notre intention, au cours d'une étude exploratoire comme celleci, de discuter de l'impact de tel ou tel type de transaction sur l'économie du comté de Berthier. Cependant, nous pouvons nous demander si la persistance de ce type de transaction n'est pas une indication d'un changement graduel qui se manifeste à cette époque dans le modèle d'émigration du comté de Berthier vers la Nouvelle-Angleterre; changement qui dénote une transition d'un modèle caractérisé principalement par une émigration temporaire vers un modèle d'émigration permanente. Le fait qu'un immigrant canadien-français résidant aux ÉtatsUnis prenne la décision de vendre à un moment précis la terre qu'il possède dans sa paroisse d'origine implique une stratégie économique 
qui révèle une volonté de rester aux États-Unis et même de s'y établir définitivement. Ce fait est d'autant plus important que pour plusieurs émigrants canadiens-français, la terre représentait encore une ressource économique très précieuse sur laquelle ils pouvaient toujours compter en prévision d'un éventuel retour et d'une réinsertion dans leur province natale.

\section{GRAPHIQUE I}

Nombre de ventes de terre par année, comté de Berthier, 1875-1905, dont le vendeur réside en Nouvelle-Angleterre.

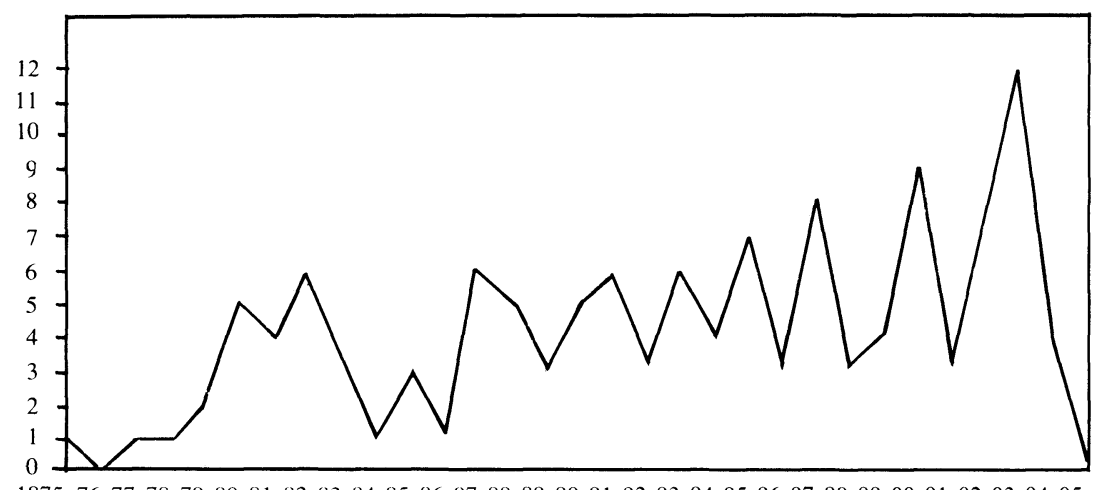

1875767778798081828384858687888990919293949596979899000102030405

Sources: Actes notariés du comté de Berthier, 1875-1905. Archives du Palais de Justice, Joliette.

Ces données sont significatives dans la mesure où le nombre de transactions, malgré les fluctuations qu'elles ont subies surtout au début de la période étudiée, augmente jusqu'en 1903 et ce, en dépit du fait que le mouvement d'émigration, à partir de la fin des années 1880 et du début des années 1890 , entre dans sa phase descendante qui se poursuivra pendant les deux premières décennies du $\mathrm{XX}^{\mathrm{e}}$ siècle.

Évidemment, d'autres recherches sont nécessaires afin de rendre cette hypothèse plus plausible. Néanmoins, il faut se rappeler les conclusions auxquelles sont parvenues certaines études ${ }^{14}$ et qui indiquaient que le mouvement d'émigration des Canadiens français au cours des années 1890 avait atteint une certaine stabilité et que les émigrants canadiens-français percevaient de plus en plus le phénomène de l'émi-

14 Voir Haebler, op. cit.; Bruno Ramirez, «A Socioeconomic Profile of the French Canadian Labour Force in the New England Cotton Industry During the Progressive Era», Labour/Le Travailleur, 11 (printemps 1983): 125-142; Bruno Ramirez, "French Canadian Immigrants in New England and their Geographical Mobility: Some Historical Hypotheses» (non publié, Université de Montréal, 1983). 
gration comme une stratégie à long terme plutôt que comme une solution temporaire à leurs problèmes immédiats.

Les données statistiques disponibles ne nous permettent pas d'établir si le mouvement d'émigration en provenance du comté de Berthier est conforme à la tendance générale suivie par le mouvement d'exode pour tout le Québec à cette époque. Mais la persistance des ventes de terre par des ex-résidents de Berthier, associée à l'émergence de réseaux d'émigration reliant ce comté à plusieurs localités des États du Rhode Island et du Massachusetts, constitue des éléments évidents de stabilisation de ce courant migratoire.

Il faut aussi constater que des transactions dénotant une tendance inverse ont également eu lieu; nous pensons au cas où un Québécois de Berthier, résidant en Nouvelle-Angleterre, achetait une terre dans son comté d'origine, ou bien louait sa terre, ce qui suggère que l'immigrant en question considérait sa résidence aux États-Unis comme étant temporaire. Cependant, notre recherche dans les actes notariés du comté de Berthier a révélé que ces types de transactions étaient très rares, ce qui confirme notre hypothèse voulant que l'émigration permanente ou à long terme était la tendance principale à l'intérieur de ce courant migratoire particulier.

Finalement, il faut souligner que les États de la Nouvelle-Angleterre et les autres régions des États-Unis ne furent pas les seules destinations choisies par les émigrants canadiens-français. Nous savons, en fait, que des centres urbains et industrialisés comme Montréal ont accueilli aussi leur contingent de migrants provenant des régions rurales du Québec. Cependant, nous connaissons bien peu de choses au sujet de la relation entre ces deux types de destination. Nous ignorons, par exemple, si l'attraction exercée sur le Québec rural par certaines régions telles le Rhode Island et Montréal fit naître deux courants migratoires différents, chacun répondant à une stratégie et à une relation particulière vis-à-vis du lieu de destination. Nous ignorons également si la population rurale qui décidait de migrer vers Montréal était constituée de gens ayant vécu une émigration vers les États-Unis ${ }^{15}$. Quoi qu'il en soit, il est certain que l'identification des réseaux précis reliant les localités du Québec et des États-Unis permet d'établir dans quelle mesure ils ont donné à ce mouvement d'émigration une configuration géo-économique et temporelle particulière. Cette connaissance aidera aussi à mieux comprendre le processus d'urbanisation qui s'est manifesté au sein de la société québécoise elle-même.

15 Dans ce contexte, l'article de Jean-Claude Robert «Urbanisation et population. Le cas de Montréal en 1861", dans la Revue d' histoire de l'Amérique française, 35, 4 (mars 1982): 523535 , est très pertinent. Bien que son texte porte principalement sur la migration des Canadiens français vers Montréal durant la période précédant 1861, l'auteur souligne, d'une manière bien concrète, l'importance d'étudier la relation entre les courants migratoires vers les États-Unis et ceux à l'intérieur du Québec. 